\title{
Expectations Confounded: Patients Are Satisfied with THR and TKR Delivery in Australian Public Hospitals-A Multisite Case Study
}

\author{
Julie Lynette Walters, Shylie Mackintosh, and Lorraine Sheppard \\ Division of Health Sciences, University of South Australia, North Terrace, Adelaide, SA 5000, Australia \\ Correspondence should be addressed to Julie Lynette Walters, julie.walters@unisa.edu.au \\ Received 30 September 2012; Accepted 1 November 2012 \\ Academic Editors: M. F. Allam, A. Bramesfeld, and A. Slep
}

Copyright ( 2012 Julie Lynette Walters et al. This is an open access article distributed under the Creative Commons Attribution License, which permits unrestricted use, distribution, and reproduction in any medium, provided the original work is properly cited.

Background. The popular media infer that satisfaction with waiting lists for total hip or knee replacement surgery is poor; however, there is little supporting evidence for this. The purpose of this study was to describe patients satisfaction with the service they received for joint replacement surgery in a public hospital. Methods. A cross-sectional design was used to survey 450 patients. Data was analysed descriptively and chi-squared analyses (significance level of $P<0.05$ ) were used to examine associations between variables. Results. The response rate was $71 \%$. Nearly three-quarters $(n=312)$ of the sample were "very happy" with the service and $92.0 \%(n=379)$ indicated that they would have their surgery again. Satisfaction was significantly associated with waiting time to the first appointment (chi-square; $P=0.002$ ), whether the patients enquired about their waiting time (chisquare; $P=0.003$ ), and the number of preoperative appointments (chisquare; $P=0.04$ ). Most participants reported a maximum acceptable waiting period for initial appointment of less than 6 months but nearly half waited longer. Conclusions. The perception that patients are unhappy with elective surgery services in Australia may be unfounded despite many waiting longer than they consider appropriate.

\section{Introduction}

There is emotive discussion in the popular press and the health literature surrounding the provision of elective surgery in publicly funded health systems in western countries $[1,2]$. This is particularly related to the length of waiting lists, despite the current evidence regarding the clinical impact of long waiting lists being ambiguous-some studies suggest that patients deteriorate over time [3-6], while others report no change in clinical status. In Australia and elsewhere, total hip (THR) and total knee replacement (TKR) surgery are commonly used procedures in the treatment of end-stage osteoarthritis $[7,8]$. Because of their commonality, they receive considerable attention and their waiting lists are easily compared to the national standards and are therefore convenient measures of how successfully the government or health service is delivering health care. Whether or not this is a fair assessment, the focus on waiting lists has encouraged governments and hospitals to introduce processes to monitor and manage waiting lists for publicly funded health services. For example, in 1996, New Zealand trialled "financially sustainable thresholds" for elective surgery, where health services could determine the number of elective surgeries performed based on their ability to fund them $[9,10]$. In 2004, Australia introduced the national waiting time targets for elective surgery performed in public hospitals [11]. Other countries have introduced reforms based on similar concepts and many have introduced priority scoring systems $[12,13]$ or selection criteria [14] to help improve equity of access amongst individuals waiting for surgery.

Despite the number of reforms and the anecdotally poor perception of waiting lists and elective surgery in public health systems, there is a lot we do not yet know about how they are managed and what patients' perceptions of them are. As a result of the media attention, we assume that patients are unhappy because they are faced with long waiting times, yet this may not be the case. There has been some investigation of patient perceptions of waiting time for THR and TKR 
surgery in the Canadian health system-a system which, like Australia, is supported by a taxation-based funding structure. Individuals in these studies reported maximum acceptable waiting periods ranging from 12 (SD 8.35) to 18 (SD 11.0) weeks $[15,16]$. No similar information is available within the Australian population.

The aims of this study were to describe (1) patients satisfaction with the service they received while waiting for THR and TKR surgery and (2) their views on waiting times for surgery. We hypothesised that the anecdotal perception of patient dissatisfaction would be supported and patients would report low satisfaction with the system and processes involved in delivering THR and TKR and that satisfaction would be inversely associated with the waiting time.

\section{Methods}

2.1. Study Setting. Health care in Australia is two-tiered. There is a "public" health system, available to all residents and funded through a taxation-based programme called "Medicare" and a "private" health system, where individuals or families purchase additional health insurance, the advantages of which include a greater choice of hospitals or doctors and flexibility regarding the timing of medical procedures or tests. In Australia, elective surgery is defined as that which can be "delayed for at least 24 hours," (i.e., THR surgery to treat osteoarthritis) compared to emergency surgery which "occurs in the most urgent or critical cases and needs to be performed within 24 hours" (i.e., THR to repair a fractured neck of femur) [11]. This study considers the delivery of elective THR and TKR replacement surgery in the Australian public health system. These procedures were selected because they have to be reported to the Australian Government as an indication of a hospital's achievement compared to current key performance indicators [17].

To receive THR or TKR in an Australian public hospital, all patients must first be referred to that hospital by their general practitioner (family physician), or another medical specialist. Therefore, to capture a complete patient pathway, in this study waiting time is divided into two phases-the time between being referred for a consult and the first appointment at the hospital and the time between first appointment and receipt of surgery. The need to consider these two phases has been reported in some literature [18]; however, it continues to be more common to report only the time between first appointment and surgery [19].

2.2. Study Design and Participants. This study used a crosssectional survey design to determine patient perceptions of the services they received while waiting for THR and TKR surgery in a South Australian public hospital. Ethics approval was obtained from the University of South Australia Human Research Ethics Committee and the ethics committees of the four participating hospitals.

Potential participants were identified from hospital theatre lists (operating lists) and targeted people who had received elective THR and TKR surgery between January 1 and December 31, $2008(n=979)$ at four publicly funded, metropolitan hospitals in South Australia. Exclusion criteria were revision THR or TKR surgery $(n=37)$, unicompartmental knee replacement $(n=31)$, THR or TKR due to trauma or emergency (i.e., fractured neck of femur) $(n=$ 142), placement in residential care facilities or documented poor cognition $(n=16)$, and other types of orthopaedic surgery (i.e., patellofemoral joint resurfacing) $(n=46)$. This resulted in a pool of 651 eligible participants.

2.3. Survey. In April 2009, each eligible participant $(n=651)$ was sent a letter of introduction, information sheet, a copy of the questionnaire, and a reply-paid envelope. Participants were asked to complete the questionnaire as it related to their most recent elective THR or TKR replacement. To improve the response rate, three weeks after the initial mailout, a reminder letter and second copy of the questionnaire were sent to individuals who had not responded (based on participant identification codes). Fifty-eight individuals returned two copies of the questionnaire, the second of which was excluded from data analysis.

2.3.1. Questionnaire Development and Testing. The development of the questionnaire followed a six-step process from generation of aims, development of guiding questions, determining hypotheses, generating the questionnaire outline, refining the design, and adjustment through review [20]. Closed-ended questions were used throughout the questionnaire as the aim was to examine the patients' perspective of the elective surgery system (i.e., length of waiting time, degree of satisfaction) rather than their experience of using that system. For ease of completion, the questionnaire was separated into five sections containing a series of dichotomous yes/no answers and 5-point Likert scales, concluding with two openended questions for additional comments.

This survey was part of a larger research project, the results of two earlier phases (interviews and hospital documentation analysis) informing the survey aims, hypothesis, and content. Guiding questions were generated to direct questionnaire content, and the series of more specific questions produced to answer each guiding question ultimately formed the basis of the final questionnaire [20]. These questions were organised into general topics to ensure that the questionnaire followed a logical order, beginning with less sensitive demographic information and concluding with the patients' opinions of the elective surgery system [20]. Questionnaire topics included patient demographics, prereferral treatment, expectations of the patient journey, prioritisation, and waiting time to surgery. Once sorted into topics, the questions were further revised and collapsed to reduce repetition and improve clarity.

Face validity of the questionnaire was assessed through feedback on questionnaire clarity and content from experts and patients. The initial draft of the questionnaire was developed by the principal researcher and an area health services advisory panel and reviewed by elective surgery system experts and members of the quality assurance department at one of the participating hospitals. They were asked to comment on content, clarity, and structure of 
TABLE 1: Survey participant characteristics.

\begin{tabular}{lcc}
\hline Characteristic & Frequency & Percentage \\
\hline Gender $(n=406)$ & & \\
$\quad$ Female & 248 & 61.1 \\
Male & 158 & 38.9 \\
\hline Employment status $(n=446)$ & 42 & 9.3 \\
$\quad$ Employed & 298 & 66.2 \\
Retired/pensioner & 88 & 19.6 \\
Unemployed/home duties & 22 & 6.0 \\
$\quad$ Not disclosed/other & & 46.4 \\
\hline Joint replaced $(n=450)$ & 209 & 53.6 \\
$\quad$ Hip & 241 & \\
$\quad$ Knee & & 73.50 \\
\hline Relative socioeconomic disadvantage & & 26.9 \\
(based on suburb of residence; $n=434)$ & 319 & \\
Decile 1-5 (most disadvantaged) & 117 & \\
Decile 6-10 (least disadvantaged) & & \\
\end{tabular}

the questionnaire and to provide feedback relating to areas which may be difficult for participants to complete. Changes were made to improve clarity, reduce overall length, and improve ease of completion, and individuals were given a second opportunity to comment.

The revised questionnaire was reviewed by five individuals of a similar age as the expected participants [21] who had osteoarthritis of the hip or knee. These individuals were recruited via convenience sampling and were asked to comment on the clarity of the questions and how simple or difficult they found it to complete the questionnaire. After discussion with these individuals, the questionnaire was restructured so that the five sections were more even in length and the wording of some questions was clarified.

2.4. Data Analysis. Data were entered into an Excel (Microsoft) spreadsheet, checked and cleaned, then transferred to PASW (version 18.0, IBM) for analysis.

Sample characteristics including height, weight, gender, employment status, and relative socioeconomic disadvantage (based on suburb of residence and 2006 Australian census data) were compared between participants from the four hospitals and between THR or TKR groups using two-tailed independent $t$-tests for continuous variables (Bonferroni adjustments to $P$ values were made to allow for multiple comparisons) and chi-squared tests were applied for categorical variables. No significant differences were observed between the participants grouped by hospital and so data were pooled for subsequent analysis. Descriptive analysis using frequency counts and percentage was used to examine responses for most questions. The two open-ended questions in section five were analysed using thematic analysis [22]. Chi-squared analysis (significance level of $P<0.05$ ) was used to examine associations between variables (such as waiting time and satisfaction) where appropriate.

\section{Results}

Of the 464 questionnaires returned (response rate $=71.4 \%$ ), 14 were excluded. Six individuals had undergone subsequent THR or TKR surgery in 2009, two had a joint replacement following trauma, and six had undergone revision THR or TKR, leaving 450 questionnaires for analysis. Sample characteristics are outlined in Table 1. The participants had a mean age of 70 years $(S D=9.13)$, with most participants being females who were retired or pensioners. Approximately an equal number of participants had THR or TKR. No information was available regarding nonresponders, so it is not possible to determine whether a systematic difference existed between the two groups.

3.1. Waiting Time. In this study, questions were asked about two aspects of waiting times: (1) the time between being referred for surgery and the first appointment at the hospital and (2) the time between first appointment and receipt of surgery (Table 2). For both of these waiting times patients were asked the following questions: how long did you think you would wait; how long did you actually wait; and what do you think is the maximum time someone should wait?

Most participants thought that the maximum acceptable waiting time for an initial appointment with a surgeon after referral was less than or equal to 6 months $(84.5 \%$ participants). A number of people clearly waited longer than this as $38 \%$ of participants reported actually waiting longer than 6 months, and seven percent reported waiting longer than two years. Similarly for the time between first appointment and surgery most participants (73.7\%) thought the maximum acceptable waiting time was less than or equal to 6 months, but only $40.7 \%$ of participants reported this to be their actual waiting time with $10 \%$ reporting waiting for longer than two years. 
TABLE 2: Reported waiting time and perceived maximum acceptable waiting times.

\begin{tabular}{|c|c|c|c|c|c|c|}
\hline \multirow{2}{*}{$\begin{array}{l}\text { Length of } \\
\text { wait } \\
\text { (months) }\end{array}$} & \multicolumn{2}{|c|}{ Expected } & \multicolumn{2}{|c|}{ Actual } & \multicolumn{2}{|c|}{ Maximum (acceptable) } \\
\hline & $\begin{array}{c}\text { 1st consult } \\
n(\%)\end{array}$ & $\begin{array}{c}\text { Surgery } \\
n(\%)\end{array}$ & $\begin{array}{c}1 \text { st consult } \\
n(\%)\end{array}$ & $\begin{array}{c}\text { Surgery } \\
n(\%)\end{array}$ & $\begin{array}{c}1 \text { st consult } \\
n(\%)\end{array}$ & $\begin{array}{c}\text { Surgery } \\
n(\%)\end{array}$ \\
\hline$\leq 6$ & $197(46.0)$ & $138(32.6)$ & $267(62.0)$ & $173(40.7)$ & $361(84.5)$ & $298(73.7)$ \\
\hline$>6-12$ & $103(24.1)$ & $130(30.7)$ & $70(16.2)$ & $99(23.3)$ & $55(12.9)$ & $97(24.0)$ \\
\hline$>12-18$ & $77(18.0)$ & $83(19.6)$ & $63(14.6)$ & $77(18.1)$ & $8(1.9)$ & $7(1.7)$ \\
\hline$>18-24$ & $0(0)$ & $41(9.7)$ & $0(0)$ & $32(7.5)$ & $2(0.5)$ & $1(0.2)$ \\
\hline$>24$ & $51(11.9)$ & $31(7.3)$ & $31(7.2)$ & $44(10.4)$ & $1(0.2)$ & $1(0.2)$ \\
\hline
\end{tabular}

1st consult: time between referral and first appointment with the surgeon.

Surgery: time between first appointment and THR/TKR surgery.

There was some mismatch between expected, actual, and maximum acceptable times. In general maximum acceptable waiting times were shorter than either expected or actual waiting times. Interestingly a smaller proportion of participants had expected waiting times of less than six months than the self-reported actual waiting times (Table 2).

3.2. Patient Satisfaction. Participants were asked two questions directly related to satisfaction: (1) overall, how happy were you with the service you received from the hospital and (2) if you could start over, would you have your hip/knee replaced?

Nearly three-quarters $(n=312)$ reported being "very happy" with the service and only 5.2 per cent reported any degree of dissatisfaction (Table 3). Given this high level of satisfaction, it was not surprising that nearly all participants $(92.0 \%, n=379)$ indicated that they would have their surgery again if given the chance to start over.

Overall satisfaction was significantly associated with three factors: (1) reported actual waiting time to first appointment with the surgeon $\left(\chi^{2} ; P=0.002\right)$, (2) whether the patient enquired about their waiting time prior to their first appointment with the surgeon $\left(\chi^{2} ; P=0.003\right)$, and (3) the number of preoperative appointments $\left(\chi^{2} ; P=0.04\right)$.

Not surprisingly, participants with the shortest waiting period for their first appointment were more satisfied overall compared with participants who experienced a longer wait. Similarly, individuals who reported contacting the hospital experienced a shorter waiting period for the first appointment than those who did make waiting time enquiries. This is possibly a function of long waiting periods instigating enquiries, rather than long waiting periods resulting from enquiries. However, this must be considered with some caution given that overall, the sample did not appear to wait for as long as expected for surgery (Table 2).

In this sample of THR and TKR recipients, individuals who had fewer pre-operative appointments were more satisfied than those with multiple pre-operative appointments, perhaps related to the existence of comorbidities and the associated increase in preparation for surgery.

Only $50 \%$ of participants completed the open-ended questions. These questions provided some, albeit limited, insight into satisfaction. Although many participants reported that waiting times for surgery were too long, some
TABLE 3: Reported level of satisfaction with the services provided by the elective surgery system.

\begin{tabular}{lc}
\hline & $\begin{array}{c}\text { Frequency }(n=427) \\
n(\%)\end{array}$ \\
\hline Very happy & $312(73.1)$ \\
Somewhat happy & $73(17.1)$ \\
Neither happy nor & $20(4.7)$ \\
unhappy & $11(2.6)$ \\
Not happy & $11(2.6)$ \\
Very unhappy & \\
\hline
\end{tabular}

also felt that public patients should expect long waiting periods:

“... all in all I feel that if I'm going in as a public patient, the I expect to have to wait for surgery...."

Along similar lines, a number of participants coveted a sense of gratitude at receiving joint replacement without an associated cost. Others, who reported dissatisfaction with the service, tended to single out issues related to disorganisation with discharge planning or a perceived lack of care.

"When I was discharged I was sent home in a community bus, sitting on a low seat for $120 \mathrm{~km}$, three days after the operation."

\section{Discussion}

This study aimed to describe (1) patient satisfaction with the service they received while waiting for THR and TKR surgery and (2) the patients' views of waiting times.

4.1. Strengths and Limitations. A major strength of this study was the high response rate $(71 \%)$ suggesting that the results are reflective of the perceptions of the South Australian population undergoing THR or TKR surgery in South Australia. Although these results must be extrapolated cautiously to earlier and subsequent cohorts of patients, there have been no significant changes in elective surgery policy in South Australia since the period of data collection.

A limitation of this study was the inability to conduct the survey prospectively. The data management systems of 
the participating hospitals made it impossible to identify eligible survey participants prior to their surgery. The retrospective nature of the data means that patient satisfaction must be interpreted with a degree of caution. Although participants were asked about their satisfaction with the service they received, it is impossible to separate this with the clinical outcomes of their surgery. Similarly, the time between undergoing THR or TKR and ultimately reporting satisfaction may have increased the risk of recall bias. A further limitation was the inability to compare responders and nonresponders for systematic differences, which may have skewed the results, presenting an erroneous overall degree of patient satisfaction.

4.2. Commentary and Implications. In general, patients were very satisfied with the service they had received: the majority of participants $(73 \%)$ reported being very happy with the service; only $5 \%$ reporting any dissatisfaction. Patients' views of acceptable waiting times did not completely match their recall of their own waiting times. Overall, participants reported waiting for less time than they expected to but longer than they felt appropriate for both their initial consultation and, subsequently, for surgery.

The research hypothesis was that the anecdotal perception of patient dissatisfaction would be supported and patients would report low satisfaction with the system and processes involved in delivering THR and TKR. This hypothesis was refuted, and patients reported an unexpectedly high satisfaction despite the poor public image of waiting lists for public hospital services $[2,23]$. We also hypothesised that satisfaction would be inversely associated with waiting time; however, an association was only found for waiting time to initial consultation. This varies from some recent work in Canada where longer than expected waiting time to surgery increased the odds of dissatisfaction [24].

Patient satisfaction was significantly associated with (1) reported waiting time to first appointment with the surgeon, (2) whether the patient enquired about their waiting time prior to their first appointment, and (3) the number of preoperative appointments. Yet these three issues were all related to the time spent waiting for first consultation, indicating a difference between patients' views and current government policy which focuses on the immediate pre-operative phase [19].

In addition to a high level of satisfaction with the service they received while waiting for THR and TKR surgery, 92\% of this sample reported that they would have their surgery again if they were given the chance to start over. This suggests that not only were patients happy with the services they received but also that the clinical outcomes of their THR or TKR were satisfactory 12 months after surgery. Satisfaction in this case could therefore be considered a global measure, which has combined the two elements of clinical outcome and system performance.

Given the overall high positive perception of both system performance and clinical outcomes reported by this group of THR and TKR recipients, it may be that reducing waiting time to surgery is not as an urgent issue as it is widely considered to be. There may be time to dedicate greater attention to ensure that accurate and representative data is collected $[25,26]$, which has the potential to inform longterm reductions in waiting times and improved access to THR or TKR surgery in western countries.

\section{Conclusion}

The results of this study indicate that the widespread assumption that patients are unhappy with elective surgery services in Australia may be unfounded. Not only were participants in this study satisfied with the service they received while waiting for THR and TKR, they displayed a degree of altruism consistent with other patient groups [27-30]. Patient satisfaction was found to be associated with the period of waiting for an initial consultation, which to date has been overlooked in the establishment of waiting time targets in Australia. It is recommended that future system reviews of THR and TKR surgery measure waiting time for initial consult and government policy be reviewed to consider the total postreferral waiting period.

\section{Ethical Approval}

This paper received approval from the University of South Australia, Royal Adelaide Hospital, Modbury Hospital, and Qeen Elizabeth Hospital Human Research Ethics Committees.

\section{Conflict of Interests}

The authors declare that they have no conflict of interests.

\section{Acknowledgments}

The authors would like to acknowledge the contribution of Associate Professor Marie Williams, Mrs. Wendy Dolejs, and Ms. Kae Martin to this work. This paper was funded by the Central Northern Adelaide Health Service.

\section{References}

[1] N. Hunter, "Shock 40\% increase in waiting times," Irish Independent, 2011.

[2] A. Styles, " $\$ 2 \mathrm{~m}$ less on hospital spending after elective surgery target missed," Sydney Morning Herald, 2011.

[3] F. Desmeules, C. E. Dionne, É. Belzile, R. Bourbonnais, and P. Frémont, "The burden of wait for knee replacement surgery: effects on pain, function and health-related quality of life at the time of surgery," Rheumatology, vol. 49, no. 5, pp. 945-954, 2010.

[4] J. M. Fielden, J. M. Cumming, J. G. Horne, P. A. Devane, A. Slack, and L. M. Gallagher, "Waiting for hip arthroplasty: economic costs and health outcomes," Journal of Arthroplasty, vol. 20, no. 8, pp. 990-997, 2005.

[5] D. S. Garbuz, M. Xu, C. P. Duncan, B. A. Masri, and B. Sobolev, "Delays worsen quality of life outcome of primary total hip arthroplasty," Clinical Orthopaedics and Related Research, no. 447, pp. 79-84, 2006. 
[6] T. J. Hoogeboom, C. H. M. van den Ende, G. van der Sluis et al., "The impact of waiting for total joint replacement on pain and functional status: a systematic review," Osteoarthritis and Cartilage, vol. 17, no. 11, pp. 1420-1427, 2009.

[7] American Academy of Orthopaedic Surgeons, "Osteoarthritis of the hip: a compendium of evidence-based information and resources," 1:1-G-5, 2003.

[8] American Academy of Orthopaedic Surgeons, "Osteoarthritis of the knee: a compendium of evidence-based information and resources," 1:1-4-18, 2002.

[9] K. Dew, J. Cumming, D. McLeod et al., "Explicit rationing of elective services: implementing the New Zealand reforms," Health Policy, vol. 74, no. 1, pp. 1-12, 2005.

[10] A. D. MacCormick and B. R. Parry, "Waiting time thresholds: are they appropriate?" ANZ Journal of Surgery, vol. 73, no. 11, pp. 926-928, 2003.

[11] Australian Government Department of Health and Ageing, "The state of our public hospitals," Tech. Rep. 1:i-84, 2008.

[12] R. Kingston, M. Carey, and E. Masterson, "Needs-based waiting lists for hip and knee arthroplasty," Irish Journal of Medical Science, vol. 169, no. 2, pp. 125-126, 2000.

[13] A. Lack, R. T. Edwards, and A. Boland, "Weights for waits: lessons from Salisbury," Journal of Health Services Research and Policy, vol. 5, no. 2, pp. 83-88, 2000.

[14] C. D. Naylor and J. I. Williams, "Primary hip and knee replacement surgery: Ontario criteria for case selection and surgical priority," Quality and Safety in Health Care, vol. 5, no. 1, pp. 20-30, 1996.

[15] B. Conner-Spady, G. Johnston, C. Sanmartin, J. J. McGurran, and T. W. Noseworthy, "Patient and surgeon views on maximum acceptable waiting times for joint replacement," Healthcare Policy, vol. 3, no. 2, pp. 102-116, 2007.

[16] B. Conner-Spady, A. Estey, G. Arnett et al., "Determinants of patient and surgeon perspectives on maximum acceptable waiting times for hip and knee arthroplasty," Journal of Health Services Research and Policy, vol. 10, no. 2, pp. 84-90, 2005.

[17] Australian Government Department of Health and Ageing, "The state of our public hospitals," Tech. Rep. 1:i-102, 2009.

[18] T. Smith, "Waiting times: monitoring the total postreferral wait," British Medical Journal, vol. 309, no. 6954, pp. 593-596, 1994.

[19] South Australian Department of Health, Policy Framework and Associated Guidelines for Elective Surgery Services, vol. 1, 2006.

[20] L. G. Portney and M. P. Watkins, Foundations of Clinical Research: Applications to Practice, Prentice-Hall, NJ, USA, 2nd edition, 2000.

[21] J. Glover, D. Hetzel, L. Glover, A. Page, and K. Leahy, Central Northern Adelaide Health Service: A Social Health Atlas, 2005.

[22] V. Braun and V. Clarke, "Using thematic analysis in psychology," Qualitative Research in Psychology, vol. 3, no. 2, pp. 77101, 2006.

[23] S. Ryan, "Longer waits for surgery put pressure on PM," The Australian, 2009.

[24] B. L. Conner-Spady, C. Sanmartin, G. H. Johnston, J. J. McGurran, M. Kehler, and T. W. Noseworthy, "The importance of patient expectations as a determinant of satisfaction with waiting times for hip and knee replacement surgery," Health Policy, vol. 101, no. 3, pp. 245-252, 2011.

[25] D. A. Cromwell and D. A. Griffiths, "Waiting time information services: how well do different statistics forecast a patient's wait?” Australian Health Review, vol. 25, no. 6, pp. 75-85, 2002.

[26] D. Cromwell and D. Griffiths, "Waiting time information services: what are the implications of waiting list behaviour for their design?" Australian Health Review, vol. 25, no. 4, pp. 40-49, 2002.

[27] B. L. Conner-Spady, G. H. Johnston, C. Sanmartin et al., "A bird can't fly on one wing: patient views on waiting for hip and knee replacement surgery," Health Expectations, vol. 10, no. 2, pp. 108-116, 2007.

[28] B. Conner-Spady, C. Sanmartin, G. Johnston, J. McGurran, M. Kehler, and T. Noseworthy, "'There are too many of us to fix.' Patients' views of acceptable waiting times for hip and knee replacement," Journal of Health Services Research and Policy, vol. 14, no. 4, pp. 212-218, 2009.

[29] R. T. Edwards, A. Boland, C. Wilkinson, D. Cohen, and J. Williams, "Clinical and lay preferences for the explicit prioritisation of elective waiting lists: survey evidence from Wales," Health Policy, vol. 63, no. 3, pp. 229-237, 2003.

[30] G. M. Woolhead, J. L. Donovan, J. A. Chard, and P. A. Dieppe, "Who should have priority for a knee joint replacement?" Rheumatology, vol. 41, no. 4, pp. 390-394, 2002. 


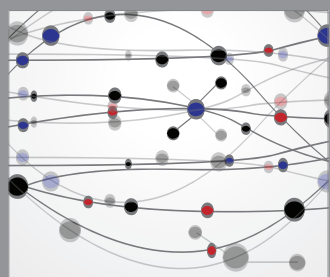

The Scientific World Journal
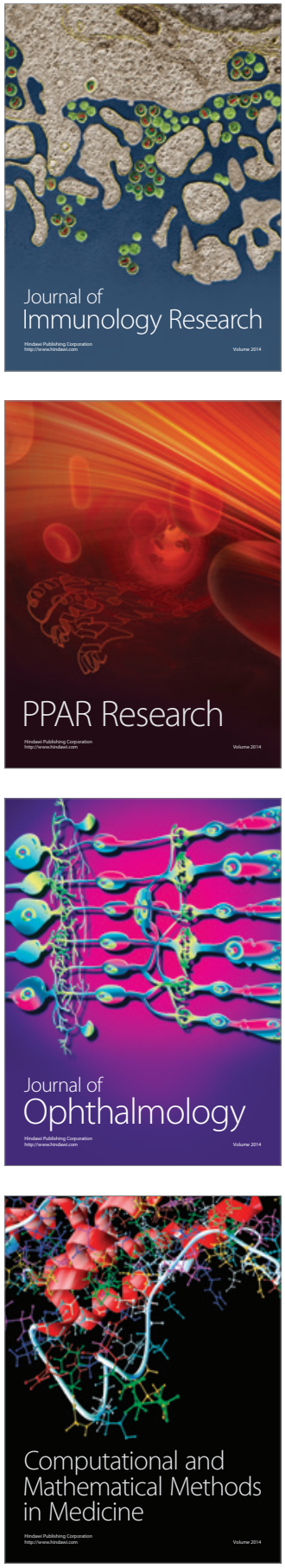

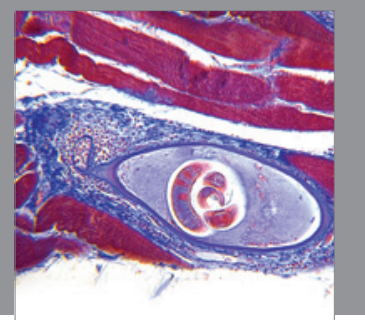

Gastroenterology

Research and Practice
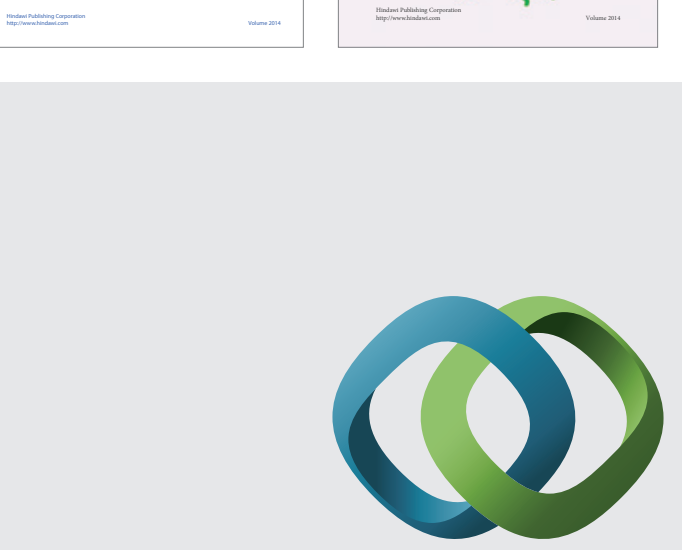

\section{Hindawi}

Submit your manuscripts at

http://www.hindawi.com
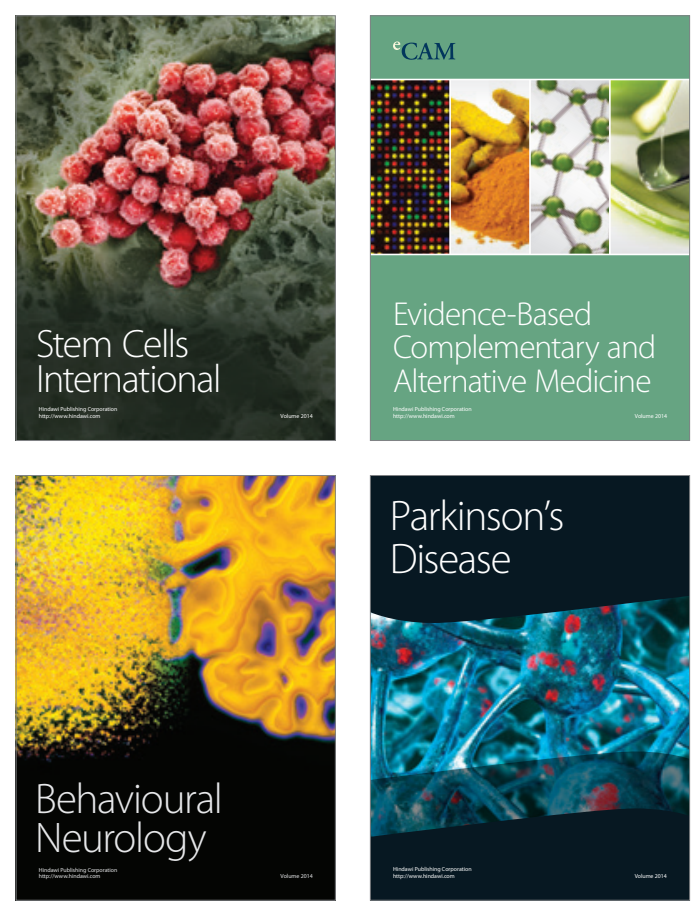

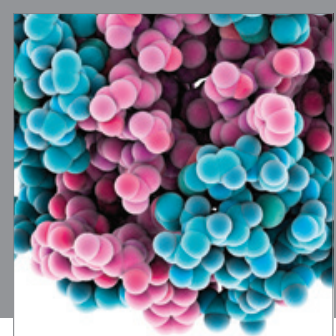

Journal of
Diabetes Research

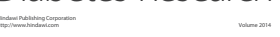

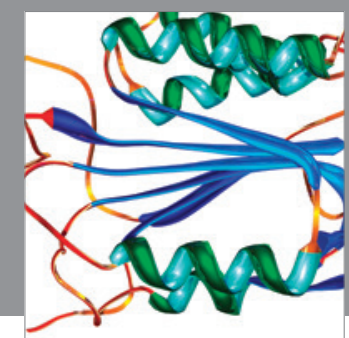

Disease Markers
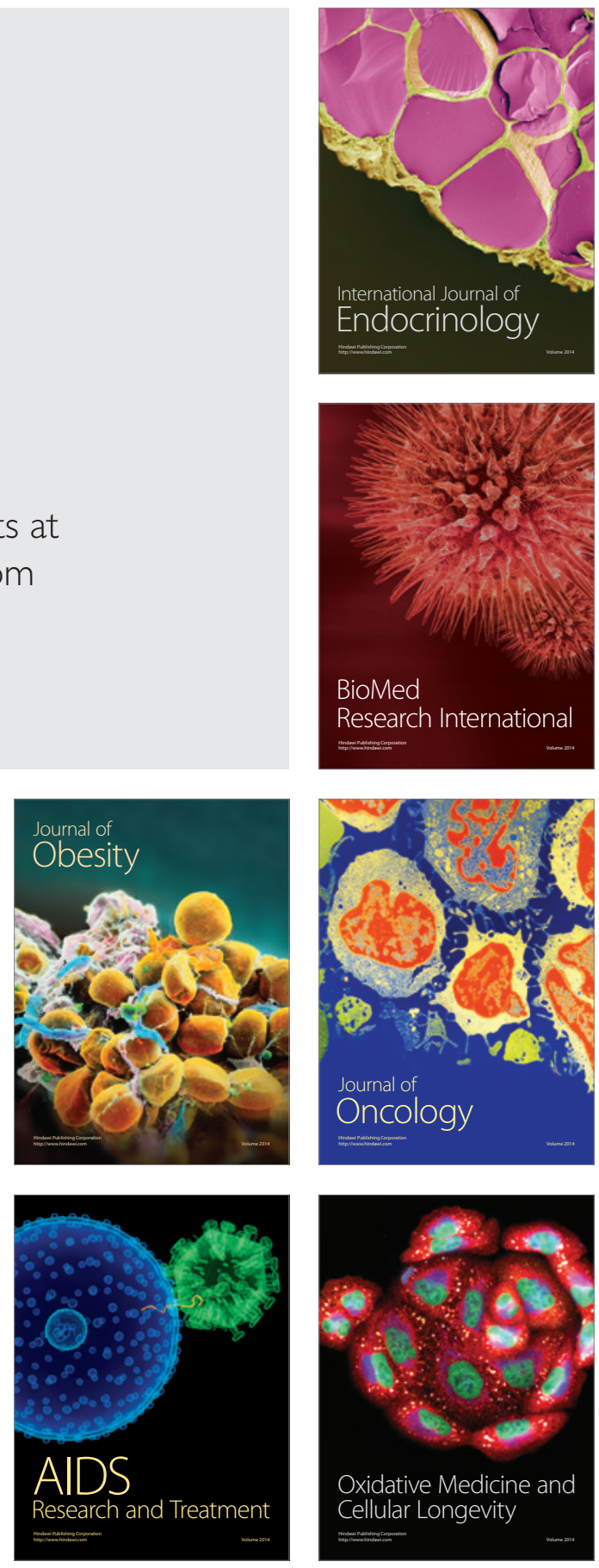